\title{
Advantage and Disadvantage of Dispersal in Two-Species Competition Models
}

\author{
Michael Winkler ${ }^{1}$ and Yuan Lou ${ }^{2, *}$ \\ ${ }^{1}$ Institut für Mathematik, Universität Paderborn, 33098 Paderborn, Germany. \\ 2 Department of Mathematics, Ohio State University, Columbus, OH 43210, USA.
}

Received 18 September 2019; Accepted 3 February 2020

\begin{abstract}
We consider a two-species competition model in which both populations are identical except their movement strategies: One species moves upward along the fitness gradient, while the other does not diffuse. While both species can coexist in homogeneous environment, we show that the species with directed movement has some advantage over the non-diffusing species in certain measurement. In contrast, if one species moves by random dispersal while the other does not diffuse, then the non-diffusing population could have advantage. Understanding the full dynamics of these ODE-PDE hybrid systems poses challenging mathematical questions.
\end{abstract}

AMS subject classifications: 35K57, 35Q92, 92D25

Key words: Competition, diffusion, population dynamics.

\section{Introduction}

An important question in spatial ecology is whether dispersal could convey some ecological or evolutionary advantages $[1,7,17]$. While random dispersal is selected against in spatially varying but temporally constant environment $[9,11,16]$, there are plenty of examples which illustrate that dispersal can be favored $[3,13,14]$. For instance, it was shown in [18] that for a two-species competition model in which both species are subject to random dispersal and passive drift, the species with the larger dispersal rate could always outcompete the species with the smaller dispersal rate, resulting the evolution of faster dispersal; See also [15,21,22] for further developments.

The main aim of this paper is to consider a deceptively simply looking scenario, in which two competing populations are identical in their competitive strengths but differ in their dispersal strategies in the following way: One species adopts certain pattern of

\footnotetext{
*Corresponding author. Email addresses: michael.winkler@math.uni-paderborn.de (M. Winkler),
} lou@math. ohio-state.edu (Y. Lou) 
dispersal but the other simply does not move. We ask: Will dispersal be advantageous or disadvantageous? To this end, we first consider

$$
\begin{cases}u_{t}=-\nabla \cdot(u \nabla(m-u-v))+u(m-u-v), & x \in \Omega, t>0, \\ v_{t}=v(m-u-v), & x \in \Omega, t>0, \\ u \cdot \frac{\partial(m-u-v)}{\partial v}=0, & x \in \partial \Omega, t>0, \\ u(x, 0)=u_{0}(x), \quad v(x, 0)=v_{0}(x), & x \in \Omega,\end{cases}
$$

in a bounded domain $\Omega \subset \mathbb{R}^{n}$ with smooth boundary $\partial \Omega$. Here $u(x, t)$ and $v(x, t)$ are densities of two populations at location $x$ and time $t . v$ denotes the outward unit normal vector on $\partial \Omega$, and the boundary condition in (1.1) means that there is no individuals of the species $u$ across $\partial \Omega$.

We envision $m$ as the available resource distribution in the habitat, and that $m-u-v$ is the effective growth rate for both populations. In fact, $m-u-v$ is often termed as the fitness of populations. In (1.1), the term $\nabla(m-u-v)$ suggests that the movement of species $u$ is solely governed by the advection upward along the fitness gradient, referred as fitness-dependent dispersal henceforth, so that the species $u$ can potentially find locations with the highest fitness in order to gain some competitive advantage. In recent years there has been increasing interest in understanding the effect of fitness-dependent dispersal on population dynamics: The single species model, i.e. (1.1) with $v \equiv 0$, was proposed by Cosner [6] and its dynamics has been studied in [8]; See also [4]. For twospecies competition model with a combination of both random and fitness-dependent movement, we refer to [5,20]; See also $[2,12]$ for other types of reaction-diffusion models with fitness-dependent dispersal.

In this paper we seek for rigorous evidence for the conjecture that the dispersive strategy in (1.1) provides the respective subpopulation with an evolutionary advantage. To see why this could be a difficult task, assume that $m$ is strictly positive in $\bar{\Omega}$. Then (1.1) has a continuum of positive steady states given by $\left\{\left(u^{*}, m-u^{*}\right)\right\}$, where $u^{*}$ satisfies $0<u^{*}(x)<m(x)$ in $\bar{\Omega}$. This implies that both populations in (1.1) could coexist. Hence, dispersal does not seem to affect the persistence of populations in this model. However, in some biological situations the total biomass of populations could also be important, e.g. populations with small size could be subject to greater danger of extinction due to stochastic effects. Therefore, a natural question will be: How does dispersal affect the relative population size, as an index of measurement of the advantage, in two competing species model (1.1)? Accordingly, throughout this paper we focus on the case

$$
m \equiv \text { const. } \quad \text { in } \Omega \text {. }
$$

We recall the following existence result from [20, Theorem 1.2]:

Theorem 1.1. Suppose that $n \geq 1$ and that $\Omega \subset \mathbb{R}^{n}$ is a bounded convex domain with smooth boundary, and $m \equiv$ const. Then for any choice of nonnegative functions $u_{0} \in \cup_{\gamma \in(0,1)} C^{\gamma}(\bar{\Omega})$ and 
$v_{0} \in W^{1, \infty}(\Omega),(1.1)$ possesses at least one global nonnegative weak solution $(u, v)$, in the sense of Definition 1.1 in [20, Theorem 1.2], such that

$$
u \in L^{\infty}(\Omega \times(0, \infty)) \cap L_{l o c}^{2}\left([0, \infty) ; W^{1,2}(\Omega)\right) \cap C^{0}\left([0, \infty) ; L^{2}(\Omega)\right)
$$

and

$$
v \in L^{\infty}(\Omega \times(0, \infty)) \cap L_{l o c}^{\infty}\left([0, \infty) ; W^{1,2}(\Omega)\right) \cap C^{0}\left([0, \infty) ; L^{2}(\Omega)\right),
$$

and $v_{t} \in L_{\text {loc }}^{2}(\bar{\Omega} \times[0, \infty))$. This solution can be obtained as the limit a.e. in $\Omega \times(0, \infty)$ of solutions to the regularized problems (2.1) below, along some sequence $\left(\varepsilon_{j}\right)_{j \in \mathbb{N}} \subset(0,1)$ such that $\varepsilon_{j} \searrow 0$ as $j \rightarrow \infty$.

As we moreover recall from [20], it is already known that solutions have the property that (in some topology), $(u(\cdot, t), v(\cdot, t)) \rightarrow\left(u_{\infty}, v_{\infty}\right)$ as $t \rightarrow \infty$, with some function $v_{\infty}$ which is positive a.e. in $\Omega$. As noted in [20], this may be somewhat surprising, because one might actually expect the first subpopulation to gain some benefit from its ability to include the dispersal mechanism expressed in (1.1) in the motion of its individuals. 1.1:

Our first goal is to derive the following feature of the solutions obtained in Theorem

Theorem 1.2. Suppose that $m \equiv$ const. and that $u_{0} \in \bigcup_{\gamma \in(0,1)} C^{\gamma}(\bar{\Omega})$ and $v_{0} \in W^{1, \infty}(\Omega)$ are such that $u_{0} \geq 0$ and $v_{0}>0$ in $\bar{\Omega}$, and let $(u, v)$ denote the corresponding solution of (1.1) from Theorem 1.1. Then

$$
\int_{\Omega} \ln \left(1+\frac{u(\cdot, t)}{v(\cdot, t)}\right) \geq \int_{\Omega} \ln \left(1+\frac{u\left(\cdot, t_{0}\right)}{v\left(\cdot, t_{0}\right)}\right)+\int_{t_{0}}^{t} \int_{\Omega} \frac{u|\nabla(u+v)|^{2}}{(u+v)^{2}} \text { for all } t_{0} \geq 0 \text { and } t>t_{0}
$$

in the sense of an inequality in $[0,+\infty]$. In particular, the functional $\int_{\Omega} \ln \left(1+\frac{u}{v}\right)$ is non-decreasing with time.

Now in order to take appropriate advantage of this property, let us introduce the following notion of exceedance in average which attempts to provide a quantitative means of measuring advantage in a way accessible to mathematical analysis:

Definition 1.1. Let $u$ and $v$ be measurable in $\Omega$ such that $u \geq 0$ and $v>0$ a.e. in $\Omega$. Then we say that $u$ exceeds $v$ in the relative average over $\Omega$ if

$$
\int_{\Omega} \frac{u}{v}>|\Omega|
$$

Within this framework, we will see that Theorem 1.2 indeed implies the following consequence:

Theorem 1.3. Suppose that $m \equiv$ const., and that $v_{0} \in W^{1, \infty}(\Omega)$ is positive in $\bar{\Omega}$ with

$$
v_{0} \not \equiv \text { const. }
$$


Then for all $T>0$ there exists $\delta(T)>0$ such that whenever $u_{0} \in \bigcup_{\gamma \in(0,1)} C^{\gamma}(\bar{\Omega})$ is nonnegative and such that

$$
v_{0} \geq u_{0} \geq v_{0}-\delta(T) \text { in } \Omega \text {, }
$$

the corresponding solution $(u, v)$ of (1.1) from Theorem 1.1 has the property that for any $t \geq T$, the function $u(\cdot, t)$ exceeds $v(\cdot, t)$ in the relative average over $\Omega$; that is, we have

$$
\int_{\Omega} \frac{u(\cdot, t)}{v(\cdot, t)}>|\Omega| \text { for all } t \geq T
$$

Remark 1.1. If two species have the same initial data and neither of them moves, then it is easy to see that $u(x, t) / v(x, t) \equiv 1$, which in particular implies

$$
\int_{\Omega} \frac{u(\cdot, t)}{v(\cdot, t)}=|\Omega| \text { for all } t \geq 0
$$

In contrast, Theorem 1.3 suggests that with the help of the biased movement upward along the fitness gradient, the species $u$ is able to increase its relative average over $v$. Moreover, by the continuous dependence of solutions on the initial data, Theorem 1.3 implies that even if initially $v$ exceeds $u$ in the relative average slightly, the species $u$ may still be able to regain its relative average over $v$ as time evolves.

This paper is organized as follows: In Section 2 we introduce a regularized problem as an approximate version of (1.1) and collect some uniform estimates on solutions to the regularized problem from [20]. Section 3 is devoted to the proof of the stable positive property of some key quantity for the approximate solutions. Various properties for certain logarithmic functionals of the solutions are established in Section 4. Theorems 1.2 and 1.3 are proved in Sections 5 and 6, respectively. In Section 7 we discuss the scenario when one species moves by random dispersal while the other does not diffuse.

\section{Preliminaries}

As an approximate version of (1.1), let us consider the regularized problem

$$
\begin{cases}\partial_{t} u_{\varepsilon}=\varepsilon \Delta u_{\varepsilon}+\nabla \cdot\left(u_{\varepsilon} \nabla\left(u_{\varepsilon}+v_{\varepsilon}-m\right)\right)+u_{\varepsilon}\left(m-u_{\varepsilon}-v_{\varepsilon}\right), & x \in \Omega, t>0, \\ \partial_{t} v_{\varepsilon}=\varepsilon \Delta v_{\varepsilon}+v_{\varepsilon}\left(m-u_{\varepsilon}-v_{\varepsilon}\right), & x \in \Omega, t>0, \\ \frac{\partial u_{\varepsilon}}{\partial v}=\frac{\partial v_{\varepsilon}}{\partial v}=0, & x \in \partial \Omega, t>0, \\ u_{\varepsilon}(x, 0)=u_{0}(x), \quad v_{\varepsilon}(x, 0)=v_{0}(x), & x \in \Omega,\end{cases}
$$

which, under the assumptions from Theorem 1.1, for each $\varepsilon \in(0,1)$ admits a globally defined classical solution $\left(u_{\varepsilon}, v_{\varepsilon}\right)$ with $u_{\varepsilon} \geq 0$ and $v_{\varepsilon}>0$ in $\bar{\Omega} \times[0, \infty)$ [20, Theorem 1.1]. 
Moreover, let us note for later reference that since we have chosen the diffusion coefficients for both $u_{\varepsilon}$ and $v_{\varepsilon}$ in (2.1) to be equal here, on addition of the respective equations we see that

$$
w_{\varepsilon}:=u_{\varepsilon}+v_{\varepsilon}, \quad \varepsilon \in(0,1),
$$

satisfies the scalar parabolic Neumann problem

$$
\begin{cases}\partial_{t} w_{\varepsilon}=\varepsilon \Delta w_{\varepsilon}+\nabla \cdot\left(u_{\varepsilon} \nabla\left(w_{\varepsilon}-m\right)\right)+w_{\varepsilon}\left(m-w_{\varepsilon}\right), & x \in \Omega, t>0, \\ \frac{\partial w_{\varepsilon}}{\partial v}=0, & x \in \partial \Omega, t>0, \\ w_{\varepsilon}(x, 0)=u_{0}(x)+v_{0}(x), & x \in \Omega .\end{cases}
$$

Apart from that, let us list here some further regularity properties of solutions to (2.1) that will be used in various places below. All these features have actually been derived in [20] already, although the dependence of the respective bounds on the initial data has not explicitly been addressed there. In order to avoid repetition of arguments, we may thus restrict ourselves to merely formulating the corresponding evident generalizations of the findings from [20] here.

The first statement in this direction, to be found in [20, Corollary 3.3], is a consequence of quite a straightforward comparison argument:

Lemma 2.1. Let $K>0$. Then there exist $C(K)>0$ and $\varepsilon_{0}(K) \in(0,1)$ such that whenever $u_{0} \in$ $\cup_{\gamma \in(0,1)} C^{\gamma}(\bar{\Omega})$ and $v_{0} \in W^{1, \infty}(\Omega)$ satisfy $u_{0} \geq 0$ and $v_{0}>0$ in $\bar{\Omega}$ and are such that

$$
\left\|u_{0}\right\|_{L^{\infty}(\Omega)}+\left\|v_{0}\right\|_{W^{1, \infty}(\Omega)} \leq K,
$$

for any choice of $\varepsilon \in\left(0, \varepsilon_{0}(K)\right)$ we have

$$
\left\|u_{\varepsilon}(\cdot, t)\right\|_{L^{\infty}(\Omega)}+\left\|v_{\varepsilon}(\cdot, t)\right\|_{L^{\infty}(\Omega)} \leq C(K) \text { for all } t>0 .
$$

We secondly collect some implications thereof, and of some appropriate testing procedures applied to (2.1), on regularity properties of first-order spatial and temporal derivatives, as deduced in [20, Lemma 3.4, Lemma 3.5 and Lemma 3.6].

Lemma 2.2. Let $K>0$, and let $\varepsilon_{0}(K) \in(0,1)$ be as provided by Lemma 2.1. Then for all $T>0$ there exists $C(K, T)>0$ such that if $0 \leq u_{0} \in \cup_{\gamma \in(0,1)} C^{\gamma}(\bar{\Omega})$ and $0<v_{0} \in W^{1, \infty}(\Omega)$ are such that (2.3) holds, we have

$$
\int_{0}^{T} \int_{\Omega}\left|\nabla u_{\varepsilon}\right|^{2} \leq C(K, T) \quad \text { for all } \varepsilon \in\left(0, \varepsilon_{0}(K)\right)
$$

and

$$
\int_{\Omega}\left|\nabla v_{\varepsilon}(\cdot, t)\right|^{2} \leq C(K, T) \quad \text { for all } t \in(0, T) \text { and any } \varepsilon \in\left(0, \varepsilon_{0}(K)\right),
$$

and moreover

and

$$
\left\|\partial_{t} u_{\varepsilon}\right\|_{L^{2}\left((0, T) ;\left(W^{1,2}(\Omega)\right)^{\star}\right)} \leq C(K, T) \quad \text { for all } \varepsilon \in\left(0, \varepsilon_{0}(K)\right)
$$

$$
\int_{0}^{T} \int_{\Omega}\left|\partial_{t} v_{\varepsilon}\right|^{2} \leq C(K, T) \quad \text { for all } \varepsilon \in\left(0, \varepsilon_{0}(K)\right)
$$




\section{A stable positivity property of $|\nabla(u+v)|$}

Lemma 3.1. Suppose that for some $T>0$ we are given two functions

$$
\tilde{u} \in L^{2}\left((0, T) ; W^{1,2}(\Omega)\right) \cap C^{0}\left([0, T] ; L^{2}(\Omega)\right)
$$

and

$$
\tilde{v} \in L^{\infty}\left((0, T) ; W^{1,2}(\Omega)\right) \cap C^{0}\left([0, T] ; L^{2}(\Omega)\right)
$$

such that $\tilde{u}$ is nonnegative as well as

$$
\tilde{u}(\cdot, 0)>0 \text { a.e. in } \Omega \text { and } \tilde{u}(\cdot, 0)+\tilde{v}(\cdot, 0) \not \equiv \text { const. }
$$

Then

$$
\int_{0}^{T} \int_{\Omega} \tilde{u}|\nabla(\tilde{u}+\tilde{v})|^{2}>0
$$

Remark 3.1. It is not claimed here that the integral in (3.1) is finite.

Proof. We divide the proof into three steps, throughout assuming that contrary to the claim of the lemma, (3.1) was false. For convenience, we write $u$ and $v$ instead of $\tilde{u}$ and $\tilde{v}$, respectively.

Step 1. We first show that there exist $c_{1}>0$ and a null set $N \subset(0, T)$ such that

$$
\int_{\Omega}|\nabla(u+v)(\cdot, t)| \leq c_{1} \quad \text { for all } t \in(0, T) \backslash N .
$$

To see this, we first note that since we assume that $\int_{0}^{T} \int_{\Omega} u|\nabla(u+v)|^{2}=0$, by nonnegativity of $u$ we obtain that $\nabla(u+v)=0$ a.e. in $\{u>0\}$. We now take $c_{1}>0$ and a null set $N \subset$ $(0, T)$ such that $u(\cdot, t)$ and $v(\cdot, t)$ both belong to $W^{1,2}(\Omega)$, that $\nabla(u+v)(\cdot, t)=0$ a.e. in $\{x \in \Omega \mid u(x, t)>0\}$ and that

$$
\int_{\Omega}|\nabla v(\cdot, t)|^{2} \leq c_{1}
$$

for all $t \in(0, T) \backslash N$, which is possible because $\nabla v \in L^{\infty}\left((0, T) ; L^{2}(\Omega)\right)$. Then for any such $t, \nabla u(\cdot, t)=0$ a.e. in $\{u(\cdot, t)=0\}:=\{x \in \Omega \mid u(x, t)=0\}$ [10, Theorem 4.4]. Therefore, whenever $t \in(0, T) \backslash N$ we have

$$
\begin{aligned}
\int_{\Omega}|\nabla(u+v)(\cdot, t)|^{2} & =\int_{\{u(\cdot, t)=0\}}|\nabla(u+v)(\cdot, t)|^{2} \\
& =\int_{\{u(\cdot, t)=0\}}|\nabla v(\cdot, t)|^{2} \\
& \leq \int_{\Omega}|\nabla v(\cdot, t)|^{2} \\
& \leq c_{1}
\end{aligned}
$$


as claimed.

Step 2. We next assert that with $N$ as above, there exists a sequence $\left(t_{k}\right)_{k \in \mathbb{N}} \subset(0, T) \backslash N$ such that $t_{k} \searrow 0$ as $k \rightarrow \infty$, that $u\left(\cdot, t_{k}\right)$ and $v\left(\cdot, t_{k}\right)$ belong to $W^{1,2}(\Omega)$ for all $k \in \mathbb{N}$, and that

$$
\nabla(u+v)\left(\cdot, t_{k}\right) \rightarrow 0 \quad \text { a.e. in } \Omega \text { as } k \rightarrow \infty .
$$

Indeed, since $u \in C^{0}\left([0, T] ; L^{2}(\Omega)\right)$, it is clear that we can find $\left(t_{k}\right)_{k \in \mathbb{N}} \subset(0, T) \backslash N$ such that $t_{k} \searrow 0$ as $k \rightarrow \infty$, that $u\left(\cdot, t_{k}\right) \in W^{1,2}(\Omega)$ and $v\left(\cdot, t_{k}\right) \in W^{1,2}(\Omega)$ with

$$
\int_{\Omega} u\left(\cdot, t_{k}\right)\left|\nabla(u+v)\left(\cdot, t_{k}\right)\right|^{2}=0 \quad \text { for all } k \in \mathbb{N},
$$

and that

$$
u\left(\cdot, t_{k}\right) \rightarrow u(\cdot, 0) \text { a.e. in } \Omega \text { as } k \rightarrow \infty .
$$

Since we have assumed $u(\cdot, 0)$ to be strictly positive a.e. in $\Omega$, we can thus pick a null set $N_{1} \subset \Omega$ such that

$$
\liminf _{k \rightarrow \infty} u\left(x, t_{k}\right)>0 \text { for all } x \in \Omega \backslash N_{1} .
$$

But by (3.3), there exists $N_{2} \subset \Omega$ such that $\left|N_{2}\right|=0$ and such that for all $k \in \mathbb{N}$,

$$
u\left(x, t_{k}\right) \cdot\left|\nabla(u+v)\left(x, t_{k}\right)\right|^{2}=0 \quad \text { for all } x \in \Omega \backslash N_{2} .
$$

Thus, combining (3.4) and (3.5) we see that for all $x \in \Omega \backslash\left(N_{1} \cup N_{2}\right)$ we can find $k_{0}(x) \in \mathbb{N}$ fulfilling

$$
\nabla(u+v)\left(x, t_{k}\right)=0 \quad \text { for all } k \geq k_{0}(x),
$$

which evidently proves (3.2).

Step 3. We finally derive a contradiction to our hypothesis $u(\cdot, 0)+v(\cdot, 0) \not \equiv$ const.

To achieve this, we take $\left(t_{k}\right)_{k \in \mathbb{N}} \subset(0, T) \backslash N$ as provided by Step 2 and then apply Step 1 to find a subsequence, still denoted by $\left(t_{k}\right)_{k \in \mathbb{N}}$ for simplicity, such that

$$
\nabla(u+v)\left(\cdot, t_{k}\right) \rightarrow z \quad \text { in } L^{2}(\Omega) \text { as } k \rightarrow \infty
$$

with some $z \in L^{2}(\Omega)$. According to Step 2 and the Egorov theorem, we must have $z=0$. On the other hand, since both $u$ and $v$ are continuous on $[0, T]$ with values in $L^{2}(\Omega)$, upon extracting another subsequence if necessary we may assume that

$$
u\left(\cdot, t_{k}\right)+v\left(\cdot, t_{k}\right) \rightarrow u(\cdot, 0)+v(\cdot, 0) \quad \text { in } L^{2}(\Omega) \text { as } k \rightarrow \infty .
$$

This, however, means that in fact $u(\cdot, 0)+v(\cdot, 0)$ belongs to $W^{1,2}(\Omega)$ and satisfies $\nabla(u+$ $v)(\cdot, 0)=0$ a.e. in $\Omega$, contradictory to our assumption on $u(\cdot, 0)+v(\cdot, 0)$. 
Lemma 3.2. Let $T>0$, and suppose that $\left(u_{k}\right)_{k \in \mathbb{N}} \subset L^{2}\left((0, T) ; W^{1,2}(\Omega)\right), u_{k} \geq 0$ for all $k \in \mathbb{N}$, and $\left(v_{k}\right)_{k \in \mathbb{N}} \subset L^{\infty}\left((0, T) ; W^{1,2}(\Omega)\right)$ are such that

$$
\left.\begin{array}{l}
\left(u_{k}\right)_{k \in \mathbb{N}} \text { is bounded in } L^{\infty}\left((\Omega \times(0, T)) \cap L^{2}\left((0, T) ; W^{1,2}(\Omega)\right),\right. \\
\left(\partial_{t} u_{k}\right)_{k \in \mathbb{N}} \text { is bounded in } L^{2}\left((0, T) ;\left(W^{1,2}(\Omega)\right)^{\star}\right),
\end{array}\right\}
$$

that

$$
\left.\begin{array}{l}
\left(v_{k}\right)_{k \in \mathbb{N}} \text { is bounded in } L^{\infty}\left((0, T) ; W^{1,2}(\Omega)\right) \\
\left(\partial_{t} v_{k}\right)_{k \in \mathbb{N}} \text { is bounded in } L^{2}\left((0, T) ;\left(W^{1,2}(\Omega)\right)^{\star}\right),
\end{array}\right\}
$$

and such that

$$
u_{k}(\cdot, 0) \rightarrow u_{0} \quad \text { and } \quad v_{k}(\cdot, 0) \rightarrow v_{0} \quad \text { in } L^{2}(\Omega) \quad \text { as } k \rightarrow \infty
$$

with certain limit functions satisfying

$$
u_{0}>0 \text { a.e. in } \Omega \text { and } u_{0}+v_{0} \not \equiv \text { const. }
$$

Then

$$
\liminf _{k \rightarrow \infty} \int_{0}^{T} \int_{\Omega} u_{k}\left|\nabla\left(u_{k}+v_{k}\right)\right|^{2}>0 .
$$

Remark 3.2. The functions $u_{k}(\cdot, 0)$ and $v_{k}(\cdot, 0)$ are well-defined, because according to a well-known result, the regularity requirements in (3.6) and (3.7) ensure that both $u_{k}$ and $v_{k}$ belong to $C^{0}\left([0, T] ; L^{2}(\Omega)\right)$ for all $k \in \mathbb{N}$.

Proof. Assuming on the contrary that (3.10) be false, on extracting a subsequence if necessary we can achieve that

$$
\int_{0}^{T} \int_{\Omega} u_{k} \cdot\left|\nabla\left(u_{k}+v_{k}\right)\right|^{2} \rightarrow 0 \text { as } k \rightarrow \infty,
$$

where in view of (3.6) and (3.7) we may also assume that

$$
\left.\begin{array}{l}
u_{k} \rightarrow \tilde{u} \quad \text { and } \quad v_{k} \rightarrow \tilde{v} \text { a.e. in } \Omega \times(0, T), \\
u_{k} \rightarrow \tilde{u} \text { in } L^{2}\left((0, T) ; W^{1,2}(\Omega)\right) \text { and } v_{k} \stackrel{\star}{\rightarrow} \tilde{v} \text { in } L^{\infty}\left((0, T) ; W^{1,2}(\Omega)\right), \\
\partial_{t} u_{k} \rightarrow \tilde{u}_{t} \text { in } L^{2}\left((0, T) ;\left(W^{1,2}(\Omega)\right)^{\star}\right) \text { and } \partial_{t} v_{k} \rightarrow \tilde{v}_{t} \quad \text { in } L^{2}\left((0, T) ;\left(W^{1,2}(\Omega)\right)^{\star}\right)
\end{array}\right\}
$$

hold as $k \rightarrow \infty$ with appropriate limit functions

$$
\left.\begin{array}{l}
\tilde{u} \in L^{\infty}(\Omega \times(0, T)) \cap L^{2}\left((0, T) ; W^{1,2}(\Omega)\right) \cap C^{0}\left([0, T] ; L^{2}(\Omega)\right), \\
\tilde{v} \in L^{\infty}\left((0, T) ; W^{1,2}(\Omega)\right) \cap C^{0}\left([0, T] ; L^{2}(\Omega)\right) .
\end{array}\right\}
$$

Clearly, $\tilde{u} \geq 0$ a.e. in $\Omega \times(0, T)$, and it is easy to see that (3.8) and (3.12) imply that

$$
\tilde{u}(\cdot, 0)=u_{0} \quad \text { and } \quad \tilde{v}(\cdot, 0)=v_{0} .
$$


We claim that moreover $\sqrt{\tilde{u}} \nabla(\tilde{u}+\tilde{v})$ belongs to $L^{2}\left(\Omega \times(0, T) ; \mathbb{R}^{n}\right)$ and in fact satisfies

$$
\int_{0}^{T} \int_{\Omega} \tilde{u}|\nabla(\tilde{u}+\tilde{v})|^{2}=0 .
$$

For this purpose, we let $z_{k}:=\sqrt{u_{k}} \nabla\left(u_{k}+v_{k}\right)$ for $k \in \mathbb{N}$ and then obtain from (3.7) that $\left(z_{k}\right)_{k \in \mathbb{N}}$ is bounded in $L^{2}(\Omega \times(0, T))$, whence passing to a subsequence we may assume that $z_{k} \rightarrow z$ in $L^{2}(\Omega \times(0, T))$ as $k \rightarrow \infty$. Since furthermore $\sqrt{u_{k}} \rightarrow \sqrt{\tilde{u}}$ in $L^{2}(\Omega \times(0, T))$ by (3.7), (3.12) and the dominated convergence theorem, and since (3.12) also entails that $\nabla\left(u_{k}+v_{k}\right) \rightarrow \nabla(\tilde{u}+\tilde{v})$ in $L^{2}(\Omega \times(0, T))$, it follows that for any fixed $\varphi \in C_{0}^{\infty}(\Omega \times(0, T))$ we have

$$
\begin{aligned}
\int_{0}^{T} \int_{\Omega} z_{k} \cdot \varphi & =\int_{0}^{T} \int_{\Omega}\left(\sqrt{u_{k}} \varphi\right) \cdot \nabla\left(u_{k}+v_{k}\right) \\
& \rightarrow \int_{0}^{T} \int_{\Omega}(\sqrt{\tilde{u}} \varphi) \cdot \nabla(\tilde{u}+\tilde{v}) \text { as } k \rightarrow \infty,
\end{aligned}
$$

which enables us to identify $z=\sqrt{\tilde{u}} \nabla(\tilde{u}+\tilde{v})$. Thus, by a lower semicontinuity argument,

$$
\begin{aligned}
\int_{0}^{T} \int_{\Omega} \tilde{u}|\nabla(\tilde{u}+\tilde{v})|^{2} & =\int_{0}^{T} \int_{\Omega}|z|^{2} \\
& \leq \liminf _{k \rightarrow \infty} \int_{0}^{T} \int_{\Omega} u_{k}\left|\nabla\left(u_{k}+v_{k}\right)\right|^{2}=0
\end{aligned}
$$

according to (3.11). In view of (3.13), (3.14) and (3.9), however, Lemma 3.1 says that (3.15) is impossible, whence in conclusion (3.10) must have been valid.

\section{The evolution of certain logarithmic functionals}

Lemma 4.1. Let $\delta \in(0,1)$. Then $w:=u+v$ satisfies

$$
\begin{aligned}
\int_{\Omega} \ln (w(\cdot, t)+\delta) \geq & \int_{\Omega} \ln \left(w\left(\cdot, t_{0}\right)+\delta\right)+\int_{t_{0}}^{t} \int_{\Omega} \frac{u}{(w+\delta)^{2}}|\nabla w|^{2} \\
& +\int_{t_{0}}^{t} \int_{\Omega} \frac{w}{w+\delta} \cdot(m-w) \quad \text { for all } t_{0} \geq 0 \text { and any } t>t_{0} .
\end{aligned}
$$

Proof. For $\varepsilon \in(0,1)$, we multiply the PDE in (2.2) satisfied by $w_{\varepsilon}=u_{\varepsilon}+v_{\varepsilon}$ by the smooth function $\frac{1}{w_{\varepsilon}+\delta}$ and integrate by parts over $\Omega$ and use that $\nabla m \equiv 0$ to find that

$$
\begin{aligned}
\frac{d}{d t} \int_{\Omega} \ln \left(w_{\varepsilon}+\delta\right)= & \varepsilon \int_{\Omega} \frac{1}{\left(w_{\varepsilon}+\delta\right)^{2}}\left|\nabla w_{\varepsilon}\right|^{2}+\int_{\Omega} \frac{u_{\varepsilon}}{\left(w_{\varepsilon}+\delta\right)^{2}}\left|\nabla w_{\varepsilon}\right|^{2} \\
& +\int_{\Omega} \frac{w_{\varepsilon}}{w_{\varepsilon}+\delta} \cdot\left(m-w_{\varepsilon}\right) \text { for all } t>0 .
\end{aligned}
$$


Since the first summand on the right is nonnegative, an integration in time yields

$$
\begin{aligned}
& \int_{\Omega} \ln \left(w_{\varepsilon}(\cdot, t)+\delta\right)-\int_{\Omega} \ln \left(w_{\varepsilon}\left(\cdot, t_{0}\right)+\delta\right)-\int_{t_{0}}^{t} \int_{\Omega} \frac{w_{\varepsilon}}{w_{\varepsilon}+\delta} \cdot\left(m-w_{\varepsilon}\right) \\
\geq & \int_{t_{0}}^{t} \int_{\Omega} \frac{u_{\varepsilon}}{\left(w_{\varepsilon}+\delta\right)^{2}}\left|\nabla w_{\varepsilon}\right|^{2} \quad \text { for all } t_{0} \geq 0 \text { and each } t>t_{0}
\end{aligned}
$$

whenever $\varepsilon \in(0,1)$. Now Lemma 2.1 says that with some $\varepsilon_{0} \in(0,1)$ and $c_{1}>0$ we have

$$
u_{\varepsilon} \leq w_{\varepsilon} \leq c_{1} \quad \text { in } \Omega \times(0, \infty)
$$

and hence

$$
\delta \leq w_{\varepsilon}+\delta \leq c_{1}+1 \quad \text { in } \Omega \times(0, \infty) .
$$

Moreover, Theorem 1.1 asserts that

$$
u_{\varepsilon} \rightarrow u \text { and } w_{\varepsilon} \rightarrow w \text { a.e. in } \Omega \times(0, \infty),
$$

whence in particular for some null set $N \subset(0, \infty)$ we have

$$
w_{\varepsilon}(\cdot, t) \rightarrow w(\cdot, t) \quad \text { a.e. in } \Omega \quad \text { for all } t \in(0, \infty) \backslash N
$$

as $\varepsilon=\varepsilon_{j} \searrow 0$. Therefore, in view of (4.3) and (4.4), the dominated convergence theorem becomes applicable to ensure that

$$
\begin{aligned}
& \int_{\Omega} \ln \left(w_{\varepsilon}(\cdot, t)+\delta\right)-\int_{\Omega} \ln \left(w_{\varepsilon}\left(\cdot, t_{0}\right)+\delta\right) \\
\rightarrow & \int_{\Omega} \ln (w(\cdot, t)+\delta)-\int_{\Omega} \ln \left(w\left(\cdot, t_{0}\right)+\delta\right) \\
& \text { for all } t_{0} \in(0, \infty) \backslash N \text { and each } t \in\left(t_{0}, \infty\right) \backslash N
\end{aligned}
$$

as well as

$$
\begin{aligned}
& \int_{t_{0}}^{t} \int_{\Omega} \frac{w_{\varepsilon}}{w_{\varepsilon}+\delta} \cdot\left(m-w_{\varepsilon}\right) \\
\rightarrow & \int_{t_{0}}^{t} \int_{\Omega} \frac{w}{w+\delta} \cdot(m-w) \text { for all } t_{0} \geq 0 \text { and any } t>t_{0}
\end{aligned}
$$

as $\varepsilon=\varepsilon_{j} \searrow 0$.

As for the integral on the right of (4.2), we argue in a more subtle way: Firstly, if $T>0$ is given then according to the above reasoning, using (4.2) we can find $c_{2}(T)>0$ such that

$$
\int_{0}^{T} \int_{\Omega} \frac{u_{\varepsilon}}{\left(w_{\varepsilon}+\delta\right)^{2}}\left|\nabla w_{\varepsilon}\right|^{2} \leq c_{2}(T)
$$


for all $\varepsilon \in\left(0, \varepsilon_{0}\right)$, whence for a subsequence $\left(\varepsilon_{j_{k}}\right)_{k \in \mathbb{N}}$ of $\left(\varepsilon_{j}\right)_{j \in \mathbb{N}}$ and some $z \in L^{2}(\Omega \times(0, T))$ we have

$$
\frac{\sqrt{u_{\varepsilon}}}{w_{\varepsilon}+\delta} \nabla w_{\varepsilon} \rightarrow z \quad \text { in } L^{2}(\Omega \times(0, T))
$$

as $\varepsilon=\varepsilon_{j_{k}} \searrow 0$. Next, since $h_{\varepsilon}:=\frac{\sqrt{u_{\varepsilon}}}{w_{\varepsilon}+\delta}$ satisfies $h_{\varepsilon} \leq \frac{\sqrt{c_{1}}}{\delta}$ in $\Omega \times(0, \infty)$, along a further subsequence, still denoted by $\left(\varepsilon_{j_{k}}\right)_{k \in \mathbb{N}}$, we have $h_{\varepsilon} \rightarrow h$ in $L^{2}(\Omega \times(0, T))$ as $\varepsilon=\varepsilon_{j_{k}} \searrow 0$. Here the observation that $h_{\varepsilon} \rightarrow \frac{\sqrt{u}}{w+\delta}$ a.e. in $\Omega \times(0, T)$ as $\varepsilon=\varepsilon_{j} \searrow 0$ by (4.5) allows us to identify $h=\frac{\sqrt{u}}{w+\delta}$, and thereupon another application of the dominated convergence theorem shows that in fact

$$
h_{\varepsilon} \rightarrow \frac{\sqrt{u}}{w+\delta} \quad \text { in } L^{2}(\Omega \times(0, T))
$$

as $\varepsilon=\varepsilon_{j_{k}} \searrow 0$. As furthermore

$$
\nabla w_{\varepsilon} \rightarrow \nabla w \quad \text { in } L^{2}(\Omega \times(0, T)) \text { as } \varepsilon=\varepsilon_{j} \searrow 0
$$

according to Theorem 1.1, this determines $z$ in (4.9): Indeed, given $\varphi \in C_{0}^{\infty}(\Omega \times(0, T))$ we may combine (4.10) and (4.11) to obtain

$$
\begin{aligned}
\int_{0}^{T} \int_{\Omega} h_{\varepsilon} \nabla w_{\varepsilon} \cdot \nabla \varphi & =\int_{0}^{T} \int_{\Omega}\left(h_{\varepsilon} \varphi\right) \cdot \nabla w_{\varepsilon} \\
& \rightarrow \int_{0}^{T} \int_{\Omega}\left(\frac{\sqrt{u}}{w+\delta} \varphi\right) \cdot \nabla w \quad \text { as } \varepsilon=\varepsilon_{j_{k}} \searrow 0
\end{aligned}
$$

which implies that $z=\frac{\sqrt{u}}{w+\delta} \nabla w$.

Now in view of lower semicontinuity of the norm in $L^{2}(\Omega \times(0, T))$ with respect to weak convergence, (4.9) in conjunction with (4.2), (4.7) and (4.8) yields

$$
\begin{aligned}
\int_{t_{0}}^{t} \int_{\Omega} \frac{u}{(w+\delta)^{2}}|\nabla w|^{2} \leq & \int_{\Omega} \ln (w(\cdot, t)+\delta)-\int_{\Omega} \ln \left(w\left(\cdot, t_{0}\right)+\delta\right) \\
& -\int_{t_{0}}^{t} \int_{\Omega} \frac{w}{w+\delta} \cdot(m-w) \\
& \text { for all } t_{0} \in(0, T) \backslash N \text { and all } t \in\left(t_{0}, T\right) \backslash N .
\end{aligned}
$$

Here we note that thanks to the mean-value theorem and the inclusion $w \in C^{0}\left([0, T] ; L^{2}(\Omega)\right)$ asserted by Theorem 1.1, the mapping $[0, T] \ni s \mapsto \int_{\Omega} \ln (w(\cdot, s)+\delta)$ is continuous, because 
for any $s$ and $s_{0}$ from $[0, T]$ we have

$$
\begin{aligned}
& \left|\int_{\Omega} \ln (w(\cdot, s)+\delta)-\int_{\Omega} \ln \left(w\left(\cdot, s_{0}\right)+\delta\right)\right|^{2} \\
\leq & |\Omega| \cdot \int_{\Omega}\left|\ln (w(\cdot, s)+\delta)-\ln \left(w\left(\cdot, s_{0}\right)+\delta\right)\right|^{2} \\
\leq & \frac{|\Omega|}{\delta^{2}} \cdot \int_{\Omega}\left|w(\cdot, s)-w\left(\cdot, s_{0}\right)\right|^{2} .
\end{aligned}
$$

Since clearly also the space-time integrals in (4.12) depend continuously on both $t_{0} \in[0, T]$ and $t \in\left(t_{0}, T\right]$, by density of $(0, T) \backslash N$ in $[0, T]$ we conclude that (4.12) implies (4.1), because $T>0$ was arbitrary.

Our analysis of the corresponding quantity involving $v$ will additionally rely on the bound on $\nabla v$ from Lemma 2.2:

Lemma 4.2. For any $\delta \in(0,1)$, we have

$$
\begin{aligned}
\int_{\Omega} \ln (v(\cdot, t)+\delta)= & \int_{\Omega} \ln \left(v\left(\cdot, t_{0}\right)+\delta\right)+\int_{t_{0}}^{t} \int_{\Omega} \frac{v}{v+\delta} \cdot(m-w) \\
& \text { for all } t_{0} \geq 0 \text { and any } t>t_{0}
\end{aligned}
$$

where $w=u+v$.

Proof. The proof parallels that of Lemma 4.1 to a certain extent, a main difference stemming from the fact that in view of the claimed exact equality in (4.13), for the diffusive term appearing in the regularization (2.1), a one-sided estimation as above is no longer sufficient here.

To be more precise, for fixed $\delta \in(0,1)$ and $T>0$ and any $\varepsilon \in(0,1)$ we multiply the second equation in (2.1) by $\frac{1}{v_{\varepsilon}+\delta}$ to see upon integrating by parts that

$$
\begin{aligned}
& \int \ln \left(v_{\mathcal{E}}(\cdot, t)+\delta\right)-\int_{\Omega} \ln \left(v_{\mathcal{\varepsilon}}\left(\cdot, t_{0}\right)+\delta\right) \\
& =\varepsilon \int_{t_{0}}^{t} \int_{\Omega} \frac{1}{\left(v_{\varepsilon}+\delta\right)^{2}}\left|\nabla v_{\varepsilon}\right|^{2}+\int_{t_{0}}^{t} \int_{\Omega} \frac{v_{\varepsilon}}{v_{\varepsilon}+\delta} \cdot\left(m-w_{\varepsilon}\right) \\
& \text { for all } t_{0} \in[0, T) \text { and any } t \in\left(t_{0}, T\right] \text {, }
\end{aligned}
$$

where $w_{\varepsilon}=u_{\varepsilon}+v_{\varepsilon}$. Since with $\varepsilon_{0}$ as in Lemma 2.1, both $\left(v_{\varepsilon}\right)_{\varepsilon \in\left(0, \varepsilon_{0}\right)}$ and $\left(w_{\varepsilon}\right)_{\varepsilon \in\left(0, \varepsilon_{0}\right)}$ are bounded in $L^{\infty}(\Omega \times(0, T))$ and, by Theorem 1.1, a.e. in $\Omega \times(0, T)$ convergent to $v$ and $w$ as $\varepsilon=\varepsilon_{j} \searrow 0$, respectively, the dominated convergence theorem guarantees that as $\varepsilon=\varepsilon_{j} \searrow 0$,

$$
\int_{t_{0}}^{t} \int_{\Omega} \frac{v_{\varepsilon}}{v_{\varepsilon}+\delta} \cdot\left(m-w_{\varepsilon}\right) \rightarrow \int_{t_{0}}^{t} \int_{\Omega} \frac{v}{v+\delta} \cdot(m-w) \quad \text { for all } t_{0} \in[0, T) \text { and } t \in\left(t_{0}, T\right]
$$


and

$$
\begin{aligned}
& \int_{\Omega} \ln \left(v_{\mathcal{E}}(\cdot, t)+\delta\right)-\int_{\Omega} \ln \left(v_{\mathcal{E}}\left(\cdot, t_{0}\right)+\delta\right) \rightarrow \int_{\Omega} \ln (v(\cdot, t)+\delta)-\int_{\Omega} \ln \left(v\left(\cdot, t_{0}\right)+\delta\right) \\
& \text { for all } t_{0} \in(0, T) \backslash N \text { and each } t \in\left(t_{0}, T\right) \backslash N
\end{aligned}
$$

with an appropriate null set $N \subset(0, \infty)$. Now since from Lemma 2.2 we know that there exists $c_{1}(T)>0$ such that

$$
\int_{\Omega}\left|\nabla v_{\mathcal{E}}(\cdot, t)\right|^{2} \leq c_{1}(T) \text { for all } t \in(0, T)
$$

whenever $\varepsilon \in\left(0, \varepsilon_{0}\right)$, the first summand on the right of (4.14) satisfies

$$
\varepsilon \int_{t_{0}}^{t} \int_{\Omega} \frac{1}{\left(v_{\mathcal{E}}+\delta\right)^{2}}\left|\nabla v_{\varepsilon}\right|^{2} \leq \frac{\varepsilon c_{1}(T) \cdot\left(t-t_{0}\right)}{\delta^{2}} \rightarrow 0 \quad \text { as } \varepsilon \searrow 0
$$

Therefore, (4.14), (4.15) and (4.16) warrant the validity of the identity in (4.13) for all $t_{0} \in(0, T) \backslash N$ and any $t \in\left(t_{0}, T\right) \backslash N$. Since the $L^{2}(\Omega)$-valued continuity of $v$ on $[0, T]$ ensures continuity of $[0, T] \ni s \mapsto \int_{\Omega} \ln (v(\cdot, s)+\delta)$, again a density argument thus yields the claim.

\section{Proof of Theorem 1.2}

Proof of Theorem 1.2. Given $t_{0} \geq 0$ and $t>t_{0}$, for $\delta \in(0,1)$ we subtract (4.13) from (4.1) to obtain

$$
\begin{aligned}
& \int_{\Omega} \ln (w(\cdot, t)+\delta)-\int_{\Omega} \ln (v(\cdot, t)+\delta) \\
\geq & \int_{\Omega} \ln \left(w\left(\cdot, t_{0}\right)+\delta\right)-\int_{\Omega} \ln \left(v\left(\cdot, t_{0}\right)+\delta\right) \\
& +\int_{t_{0}}^{t} \int_{\Omega} \frac{u}{(w+\delta)^{2}}|\nabla w|^{2}+\int_{t_{0}}^{t} \int_{\Omega}\left(\frac{w}{w+\delta}-\frac{v}{v+\delta}\right) \cdot(m-w)
\end{aligned}
$$

with $w=u+v$. Since in the pointwise sense we have

$$
\ln (w+\delta)-\ln (v+\delta)=\ln \frac{u+v+\delta}{v+\delta}=\ln \left(1+\frac{u}{v+\delta}\right) \nearrow \ln \left(1+\frac{u}{v}\right) \quad \text { a.e. in } \Omega \times\left(t_{0}, t\right)
$$

as $\delta \searrow 0$, two applications of the monotone convergence theorem yield

$$
\int_{\Omega} \ln (w(\cdot, t)+\delta)-\int_{\Omega} \ln (v(\cdot, t)+\delta) \nearrow \int_{\Omega} \ln \left(1+\frac{u(\cdot, t)}{v(\cdot, t)}\right) \in[0, \infty]
$$

and

$$
\int_{\Omega} \ln \left(w\left(\cdot, t_{0}\right)+\delta\right)-\int_{\Omega} \ln \left(v\left(\cdot, t_{0}\right)+\delta\right) \nearrow \int_{\Omega} \ln \left(1+\frac{u\left(\cdot, t_{0}\right)}{v\left(\cdot, t_{0}\right)}\right) \in[0, \infty]
$$


as $\delta \searrow 0$. By the same token,

$$
\int_{t_{0}}^{t} \int_{\Omega} \frac{u}{(w+\delta)^{2}}|\nabla w|^{2} \nearrow \int_{t_{0}}^{t} \int_{\Omega} \frac{u}{w^{2}}|\nabla w|^{2} \in[0, \infty] \quad \text { as } \delta \searrow 0 .
$$

Finally, observing that both $\frac{w}{w+\delta} \rightarrow 1$ and $\frac{v}{v+\delta} \rightarrow 1$ a.e. in $\Omega \times\left(t_{0}, t\right)$ and

$$
\left|\left(\frac{w}{w+\delta}-\frac{v}{v+\delta}\right) \cdot(m-w)\right| \leq 2\left(|m|+\|w\|_{L^{\infty}(\Omega \times(0, t))}\right) \quad \text { a.e. in } \Omega \times\left(t_{0}, t\right)
$$

for all $\delta \in(0,1)$, the dominated convergence theorem ensures that

$$
\int_{t_{0}}^{t} \int_{\Omega}\left(\frac{w}{w+\delta}-\frac{v}{v+\delta}\right) \cdot(m-w) \rightarrow 0 \quad \text { as } \delta \searrow 0 .
$$

Therefore, in view of (5.2)-(5.4), the inequality (1.5) is a consequence of (5.1).

\section{Proof of Theorem 1.3}

Proof of Theorem 1.3. We claim that for all $T>0$ there exists $\delta(T)>0$ such that whenever $u_{0} \in \bigcup_{\gamma \in(0,1)} C^{\gamma}(\bar{\Omega})$ is nonnegative and such that (1.7) holds, then the corresponding solution $(u, v)$ of $(1.1)$ from Theorem 1.1 satisfies

$$
\int_{\Omega} \ln \left(1+\frac{u(\cdot, t)}{v(\cdot, t)}\right)>|\Omega| \cdot \ln 2 \text { for all } t \geq T .
$$

Indeed, if this claim were false, then there would exist $T>0$ such that for all $k \in \mathbb{N}$ we could find some nonnegative $u_{0 k} \in \bigcup_{\gamma \in(0,1)} C^{\gamma}(\bar{\Omega})$ such that

$$
v_{0} \geq u_{0 k} \geq v_{0}-\frac{1}{k} \quad \text { in } \Omega,
$$

but such that the corresponding solutions $\left(u_{k}, v_{k}\right)$ of (1.1) emanating from $\left(u_{0 k}, v_{0}\right)$ would satisfy

$$
\int_{\Omega} \ln \left(1+\frac{u_{k}(\cdot, T)}{v_{k}(\cdot, T)}\right) \leq|\Omega| \cdot \ln 2 \text { for all } k \in \mathbb{N},
$$

where we have used that $[0, \infty) \ni t \mapsto \int_{\Omega} \ln \left(1+\frac{u_{k}(\cdot, t)}{v_{k}(\cdot, t)}\right)$ is nondecreasing by Theorem 1.2. Now since the estimates in Lemma 2.2 depend on $u_{0}$ only through its norm in $L^{\infty}(\Omega)$, in view of (6.2) it is clear from the latter that we can find $c_{1}>0$ such that for all $k \in \mathbb{N}$,

$$
\int_{0}^{T} \int_{\Omega}\left|\nabla u_{k}\right|^{2} \leq c_{1} \quad \text { and } \quad \int_{\Omega}\left|\nabla v_{k}(\cdot, t)\right|^{2} \leq c_{1} \quad \text { for all } t \in(0, T)
$$

as well as

$$
\int_{0}^{T}\left\|\partial_{t} u_{k}(\cdot, t)\right\|_{\left(W^{1,2}(\Omega)\right)^{\star}}^{2} d t \leq c_{1} \quad \text { and } \quad \int_{0}^{T} \int_{\Omega}\left|\partial_{t} v_{k}\right|^{2} \leq c_{1}
$$


As (6.2) moreover guarantees that

$$
u_{0 k} \rightarrow u_{0}:=v_{0} \quad \text { in } L^{\infty}(\Omega) \text { as } k \rightarrow \infty,
$$

where by assumption we have $u_{0}>0$ a.e. in $\Omega$ and $u_{0}+v_{0}=2 v_{0} \not \equiv$ const., Lemma 3.2 applies so as to yield $c_{2}>0$ and a subsequence, not relabeled for convenience, such that

$$
\int_{0}^{T} \int_{\Omega} u_{k}\left|\nabla\left(u_{k}+v_{k}\right)\right|^{2} \geq c_{2} \quad \text { for all } k \in \mathbb{N}
$$

Thus, the quantitative monotonicity estimate in Theorem 1.2 shows that

$$
\begin{aligned}
\int_{\Omega} \ln \left(1+\frac{u_{k}(\cdot, T)}{v_{k}(\cdot, T)}\right) & \geq \int_{\Omega} \ln \left(1+\frac{u_{0 k}}{v_{0}}\right)+\int_{0}^{T} \int_{\Omega} u_{k}\left|\nabla\left(u_{k}+v_{k}\right)\right|^{2} \\
& \geq \int_{\Omega} \ln \left(1+\frac{v_{0}-\frac{1}{k}}{v_{0}}\right)+c_{2}
\end{aligned}
$$

for all $k \in \mathbb{N}$. But the monotone convergence theorem asserts that

$$
\int_{\Omega} \ln \left(1+\frac{v_{0}-\frac{1}{k}}{v_{0}}\right) \nearrow|\Omega| \cdot \ln 2 \text { as } k \rightarrow \infty,
$$

whence

$$
\liminf _{k \rightarrow \infty} \int_{\Omega} \ln \left(1+\frac{u_{k}(\cdot, T)}{v_{k}(\cdot, T)}\right) \geq|\Omega| \cdot \ln 2+c_{2} .
$$

This contradicts (6.3) and thereby proves the claim.

By the inequality $x-1 \geq 2 \ln \frac{1+x}{2}$ for $x>-1$, it follows from (6.1) that

$$
\int_{\Omega} \frac{u(\cdot, t)}{v(\cdot, t)}-|\Omega| \geq 2 \int_{\Omega} \ln \left(1+\frac{u(\cdot, t)}{v(\cdot, t)}\right)-2|\Omega| \cdot \ln 2>0 \quad \text { for all } t \geq T,
$$

that is, (1.8) holds.

\section{On disadvantage of dispersal}

Diffusion may not always be advantageous in the sense of Definition 1.1. As an example, in this section we consider

$$
\begin{cases}u_{t}=d \Delta u+u(m-u-v), & x \in \Omega, t>0 \\ v_{t}=v(m-u-v), & x \in \Omega, t>0 \\ \frac{\partial u}{\partial v}=0, & x \in \partial \Omega, t>0 \\ u(x, 0)=u_{0}(x), \quad v(x, 0)=v_{0}(x), & x \in \Omega\end{cases}
$$


in a bounded domain $\Omega \subset \mathbb{R}^{n}$ with smooth boundary $\partial \Omega, d>0$ is a constant. In contrast with (1.1), species $u$ in (7.1) adopts random diffusion, i.e. the transition probability of the individuals moving in all directions are the same. It turns out that, in contrast to fitness-dependent diffusion in (1.1), random diffusion can be disadvantageous in various ways.

According to the result of Dockery et al. [9], if both species have instead positive random diffusion rates, the species with the smaller diffusion rate will become dominant and always drive the species with the larger diffusion rate to extinction, irrespective of the initial condition, provided that $m$ is non-constant. It might be natural to inquire that, if the diffusion rate of $v$ is equal to zero as in system (7.1), does it remain that the nodiffusing species $v$ will be dominant? Recently it has been shown in [19] that if $m(x)$ changes sign, for positive and continuous initial data, solutions of (7.1) will converge to $\left(0, m_{+}\right)$uniformly in $x$ as $t \rightarrow \infty$, where $m_{+}:=\max \{m(x), 0\}$. This implies that if the underlying environment has both sources and sinks, which correspond to the regions of positive and negative $m$, respectively, the no-diffusing species will drive the diffusing species to extinction and is thus advantageous. However, the answer is different when $m$ is positive: For strictly positive function $m$, system (7.1) has a continuum of positive steady states, given by $(C, m(x)-C)$, where $C$ is any constant satisfying $C \in\left(0, \min _{\bar{\Omega}} m\right)$. In particular, this implies that two competing populations in (7.1) may be able to coexist.

Does $v$ still have the advantage when both population can coexist? If so, can we measure the advantage of species $v$ in the sense of Definition 1.1? Assuming that the initial data $u_{0}, v_{0}$ in (7.1) have proper regularity, we can establish the following identity:

$$
\int_{\Omega} \ln \frac{v(\cdot, t)}{u(\cdot, t)}=\int_{\Omega} \ln \frac{v\left(\cdot, t_{0}\right)}{u\left(\cdot, t_{0}\right)}+d \int_{t_{0}}^{t} \int_{\Omega} \frac{|\nabla u|^{2}}{u^{2}}
$$

for all $t_{0} \geq 0$ and $t>t_{0}$; See [19]. In particular, if $v_{0} \geq u_{0}>0$ and $u_{0} \neq \equiv$ const. in $\bar{\Omega}$, then

$$
\int_{\Omega} \ln \frac{v(\cdot, t)}{u(\cdot, t)}>0 \text { for all } t>0
$$

Applying the inequality $x \geq 1+\ln x$ for $x>0$, we have

$$
\int_{\Omega} \frac{v(\cdot, t)}{u(\cdot, t)}>|\Omega| \text { for all } t>0,
$$

i.e. the corresponding solution $(u, v)$ of $(7.1)$ has the property that $v(\cdot, t)$ exceeds $u(\cdot, t)$ in the relative average over $\Omega$ for all $t>0$, in strong contrast to (1.8) which holds for the solutions of (1.1).

The conclusions from Theorem 1.3 and the discussions in this section illustrate that the global dynamics of the ODE-PDE hybrid systems (1.1) and (7.1) could be quite different from that of the kinetic system

$$
\begin{cases}u_{t}=u(m-u-v), & t>0, \\ v_{t}=v(m-u-v), & t>0, \\ u(0)=u_{0}, \quad v(0)=v_{0} . & \end{cases}
$$


Furthermore, the details of such differences could depend upon the movement strategies in subtle but meaningful ways. Many questions concerning the full dynamics of (1.1) are open, e.g., what are the large time asymptotic behaviors of the solutions to system (1.1) when function $m(x)$ is positive and non-constant? We hope to address these questions in future investigations.

\section{Acknowledgments}

We are grateful to two anonymous referees and Dr. Rachidi Salako for their helpful comments and suggestions. MW was partially supported by the German Research Foundation grants DFG-288366228 and DFG-411007140. YL was partially supported by the NSF grant DMS-1853561.

\section{References}

[1] R.S. Cantrell, C. Cosner, Spatial Ecology via Reaction-Diffusion Equations. Series in Mathematical and Computational Biology, John Wiley and Sons, Chichester, UK, 2003.

[2] R.S. Cantrell and C. Cosner, Evolutionary stability of ideal free dispersal under spatial heterogeneity andtime periodicity, Math. Biosciences 305 (2018) 71-76.

[3] R.S. Cantrell, C. Cosner, M. Lewis and Y. Lou, Evolution of dispersal in spatial population models with multiple timescales, J. Math. Biol. 80 (2020) 3-37.

[4] R.S. Cantrell, C. Cosner and Y. Lou, Approximating the ideal free distribution via reactiondiffusion-advection equations, J. Differential Equations 245 (2008) 3687-3703.

[5] R.S. Cantrell, C. Cosner, Y. Lou and C. Xie, Random dispersal versus fitness-dependent dispersal, J. Differential Equations 254 (2013) 2905-2941.

[6] C. Cosner, A dynamic model for the ideal-free distribution as a partial differential equation, Theor. Pop. Biol. 67 (2005) 101-108.

[7] C. Cosner, Reaction-diffusion-advection models for the effects and evolution of dispersal, Discre. Contin. Dyn. Syst. A 34 (2014) 1701-1745.

[8] C. Cosner and M. Winkler, Well-posedness and properties of a dynamical model for the ideal free distribution, J. Math. Biol. 69 (2014) 1343-1382.

[9] J. Dockery, V. Hutson, K. Mischaikow and M. Pernarowski, The evolution of slow dispersal rates: a reaction-diffusion model, J. Math. Biol. 37 (1998) 61-83.

[10] L.C. Evans and R.F. Gariepy, Measure Theory and Fine Properties of Functions, Revised Edition, Chapman and Hall/CRC, 2015.

[11] A. Hastings, Can spatial variation alone lead to selection for dispersal? Theor. Popul. Biol. 24 (1983) 244-251.

[12] L. Korobenko and E. Braverman, On evolutionary stability of carrying capacity driven dispersal in competition with regularly diffusing populations, J. Math. Biol. 69 (2014) 1181-1206.

[13] K.-Y. Lam and Y. Lou, Evolutionarily stable and convergent stable strategies in reactiondiffusion models for conditional dispersal, Bull. Math. Biol. 76 (2014) 261-291.

[14] K.-Y. Lam and Y. Lou, Evolution of dispersal: ESS in spatial models, J. Math. Biol. 68 (2014) 851-877.

[15] K.-Y. Lam, Y. Lou and F. Lutscher, Evolution of dispersal in closed advective environments, J. Biol. Dyn. 9 (2015) 188-212. 
[16] S.A. Levin, Population dynamic models in heterogeneous environments, Annu. Reu. Ecol. Syst. 7 (1976) 287-310.

[17] Y. Lou, Some challenging mathematical problems in evolution of dispersal and population dynamics, Tutorials in mathematical biosciences. IV, 171-205, Lecture Notes in Math. 1922, Math. Biosci. Subser., Springer, Berlin, 2008.

[18] Y. Lou and F. Lutscher, Evolution of dispersal in open advective environments, J. Math. Biol. 69 (2014) 1319-1342.

[19] Y. Lou and R. Salako, Dynamics in a parabolic-ODE competition system in heterogeneous environments, Proc. Amer. Math. Soc., to appear. DOI: https://doi.org/10.1090/proc/14972

[20] Y. Lou, Y. Tao and M. Winkler, Approaching the ideal free distribution in two-species competition models with fitness-dependent dispersal, SIAM J. Math. Anal. 46 (2014) 1228-1262.

[21] Y. Lou, D.M. Xiao and P. Zhou, Qualitative analysis for a Lotka-Volterra competition system in advective homogeneous environment, Discre. Contin. Dyn. Syst. A 36 (2016) 953-969.

[22] Y. Lou and P. Zhou, Evolution of dispersal in advective homogeneous environments: The effect of boundary conditions, J. Differential Equations 259 (2015) 141-171. 